\title{
Evaluation of MMP-2, MMP-9, TIMP-1, TIMP-2, NGAL and MMP-9/NGAL complex in urine and sera from patients with bladder cancer
}

\author{
SERENA RICCI ${ }^{1}$, DARIO BRUZZESE ${ }^{2}$ and ANGELINA DI CARLO ${ }^{3}$ \\ Departments of ${ }^{1}$ Translational Medical Science and ${ }^{2}$ Public Health, University of Naples 'Federico II', Naples 80131; \\ ${ }^{3}$ Department of Medico-Surgical Sciences and Biotechnologies, 'Sapienza' University of Rome, Latina 04100, Italy
}

Received September 24, 2014; Accepted June 22, 2015

DOI: $10.3892 / \mathrm{ol} .2015 .3558$

\begin{abstract}
The identification of biomarkers in urine or serum samples from patients with bladder cancer is urgently required for the development of non-invasive methods for the diagnosis of bladder carcinoma and to facilitate follow-up surveillance, to combat the high progression and recurrence rates of this type of cancer. The current study measured the content of matrix metalloproteinase (MMP)-2 and -9, as well as tissue inhibitor of metalloproteinase (TIMP)-1 and -2 in the urine and sera of 41 patients with bladder cancer by ELISA. The association between levels of MMP-2 and -9 and TIMP-1 and -2 , and tumor grade and stage were investigated to verify whether these molecules are involved in tumor differentiation. Statistical analysis of the data revealed that urinary TIMP-1 levels were significantly higher in the high grade group compared with those of the low grade samples $(\mathrm{P}=0.022)$. The results also revealed a significantly differing distribution of TIMP-1 expression between Ta and T1 stage specimens $(\mathrm{P}=0.040)$. The corresponding area under the curves (AUCs) were 0.72 , with a sensitivity of 0.70 and specificity of 0.75 . In addition, neutrophil gelatinase-associated lipocalin (NGAL) and MMP-9/NGAL complex levels in the sera were measured. All molecules evaluated were detected in the sera of the patients studied. In particular, tumors staged as non-muscle invasive (Ta and T1), demonstrated significantly higher NGAL levels compared with those of muscle invasive ( $>\mathrm{T} 1)$ bladder cancer (32.8 ng/ml vs. $16.2 \mathrm{ng} / \mathrm{ml} ; \mathrm{P}=0.029)$. The discriminatory ability of NGAL expression was confirmed by receiver
\end{abstract}

Correspondence to: Professor Angelina Di Carlo, Department of Medico-Surgical Sciences and Biotechnologies, 'Sapienza' University of Rome, 79 Corso della Repubblica, Latina 04100, Italy E-mail: angelina.dicarlo@uniroma1.it

Key words: matrix metalloproteinase-2, matrix metalloproteinase-9, tissue inhibitor of metalloproteinase-1, sera, tissue inhibitor of metalloproteinase-2, urine, neutrophil gelatinase-associated lipocalin, bladder carcinoma operating characteristic curve analysis that revealed an AUC of 0.75 , a sensitivity of 0.88 and a specificity of 0.67 . These data indicated that urinary TIMP-1 and serum NGAL may be useful non-invasive biomarkers to provide clinical information for bladder cancer disease management. Multicenter, prospective studies are required to confirm these preliminary results.

\section{Introduction}

Bladder cancer $(\mathrm{BCa})$ is one of the most common urologic malignancies worldwide; with a higher frequency amongst males than females (1). The two major environmental risk factors for $\mathrm{BCa}$ are high exposure to tobacco smoke, and occupational exposure to aromatic amines, polycyclic aromatic hydrocarbons and chlorinated hydrocarbons $(2,3)$. Furthermore, exposure to ionizing radiation, a variety of lifestyle choices, occupations, dietary factors, drugs, urologic pathologies, family history and genetic polymorphisms may increase the risk of bladder carcinoma (1-3). There are two clinical phenotypes of bladder carcinoma: Non-invasive and invasive BCa. Non-invasive BCa includes the papillary carcinoma phenotype (Ta) and flat carcinoma in situ (cis). Ta bladder carcinoma is superficial and rarely invades the basement membrane or metastasizes, but is associated with a high risk of local recurrence; while invasive bladder carcinoma has a high risk of disease progression, including muscle invasion and metastasis, and mortality. Ta tumorigenesis typically occurs via a molecular pathway that is distinct from that of cis and the invasive cancer phenotype (4). Superficial tumors are generally treated with transurethral tumor resection and intravesical Bacille Calmette-Guérin, whereas invasive tumors are aggressively resected (5). To date, cystoscopy remains the gold standard for the diagnosis of malignancies of the bladder. In addition, examination by cystoscopy is required not only for diagnosis, but also for follow-up. Cytoscopic examination is repeated at 3-month intervals as no other method currently available is sufficiently sensitive and specific. However, cystoscopy is an invasive, relatively costly and uncomfortable diagnostic method, which may also be inconclusive, particularly in the diagnosis of cystitis. Therefore, the development of non-invasive methods for the diagnosis of bladder carcinoma is urgently required. Urinary or circulating biomarkers 
represent a potential area of interest for the early detection and surveillance of bladder carcinoma due to the high accessibility of samples. Multiple tumor biomarkers have been investigated in this capacity, with variable results (6-9).

An essential change that occurs in malignancies is tissue invasion; however, the extracellular matrix (ECM) provides a significant barrier to tumor cell invasion. A unique class of matrix degrading enzymes, the matrix-metalloproteinases (MMPs), are able to degrade certain components of the ECM and basement membrane, facilitating tumor cell dissemination. In addition, MMPs have key functions in the maintenance of a supportive local environment that promotes tumor cell growth at the primary and metastatic sites. The ability to degrade type IV collagen, the major component of the basement membrane, is unique to MMP-2 and -9. These MMPs are frequently associated with the malignant phenotype of tumor cells, and their expression has been found to be elevated in several cases of human tumors exhibiting aggressive characteristics and low overall survival $(10,11)$. As a result of their potential and destructive nature, the activities of the MMPs are highly regulated at various levels, including via transcriptional control, secretion from cells as inactive precursors and functional inhibition by the tissue inhibitor of metalloproteinases (TIMPs), of which there are four: TIMP-1, TIMP-2, TIMP-3 and TIMP-4 (12). TIMP-1 binds and inhibits MMPs with 1:1 stoichiometry, binding MMP-9 in particular; while TIMP-2 inactivates MMP-2 specifically. Dysregulation of the balance between MMP and TIMP expression has been suggested to facilitate tumor progression and recurrence in cancer.

A previous study by our group revealed, by gelatin zymography, that urinary MMP-2 and -9 expression was correlated with increased MMP-9 lytic activity in high-grade and advanced-stage bladder cancer (13). In addition, a complex of MMP-9 and human neutrophil gelatinase-associated lipocalin (NGAL) has been detected in the urine of patients with prostate cancer and $\mathrm{BCa}$ (14). NGAL is an acute-phase protein involved in innate immunity, with a crucial role in intracellular iron transport (15). NGAL expression is altered in several benign conditions, including inflammatory, ischemic and metabolic disorders. Furthermore, NGAL is overexpressed in numerous types of tumor $(15,16)$, and its expression is associated with invasive cancer progression $(17,18)$.

In the present study, urinary and serum levels of MMP-2, MMP-9, TIMP-1 and TIMP-2, as well as serum concentrations of NGAL and MMP-9/NGAL complex were evaluated in samples from 41 patients with bladder carcinoma. These levels were compared with tumor grade and stage in order to verify whether these molecules may offer potential as non-invasive biomarkers to provide useful clinical information for bladder cancer disease management.

\section{Patients and methods}

Patients. A total of 41 patients were enrolled at the Departmtent of Urology, University of Naples (Naples, Italy) between May 2010 and 2011. Samples of first morning urine and peripheral venous blood were collected prior to surgical or other therapeutic interventions. Standard clinical laboratory criteria and histopathological investigations were used to diagnose and confirm the tumor type of each patient. Patient ages ranged from 40 to 86 years (mean, $71.1 \pm 9.3$ years; median, 72 years), and in total, there were 4 females and 37 males. The tumors were classified by grade and stage according to pTNM classifications (19). All patients included provided written informed consent and the study was approved by the ethics committee of the University of Naples. A total of 40 age-matched, normal, healthy laboratory volunteers were recruited as controls and provided their permission verbally. Healthy volunteers exhibited no sign of illness and their basic laboratory parameter values were confirmed to be within reference limits.

Urine sample preparation. The MultistixCombur test (Roche Diagnostics GmbH, Mannheim, Germany) was used to examine urine samples prior to analysis, and any urine samples that tested positive for leukocytes were excluded due to the presence of confounding leukocyte gelatinases. Microscopic hematuria, which was present in the majority of cancer samples, was not quantified; however, samples exhibiting macroscopic hematuria were excluded. The samples were frozen immediately following collection, and stored at $-20^{\circ} \mathrm{C}$ prior to analysis. For further analysis, samples were thawed and $15-\mathrm{ml}$ aliquots of each sample were centrifuged at $1,000 \mathrm{xg}$ for $10 \mathrm{~min}$ at $4^{\circ} \mathrm{C}$. The supernatant was collected and the levels of MMP-2, MMP-9, TIMP-1 and TIMP-2 were determined by immunoassay.

Serum. Peripheral venous blood samples were collected in vacutainers and allowed to clot for $30 \mathrm{~min}$ at room temperature, prior to centrifugation at $1,600 \mathrm{x} \mathrm{g}$ for $10 \mathrm{~min}$ at $4{ }^{\circ} \mathrm{C}$. The samples were then divided into aliquots and stored at $-20^{\circ} \mathrm{C}$ prior to the determination of MMP-2, MMP-9, TIMP-1, TIMP-2, NGAL and MMP-9/NGAL complex levels by immunoassay. Each aliquot was used only once, in order to prevent enzyme activation due to the freeze-thawing processes.

Measurement of MMP-2, MMP-9, TIMP-1, TIMP-2, NGAL and MMP-9/NGAL levels. MMP-2 and -9, as well as TIMP-1 and -2 levels were detected by ELISA using commercial kits (Biotrak Cell Proliferation ELISA System) obtained from GE Healthcare Life Sciences (Chalfont, UK). These assays were based on a two-site sandwich format, utilizing two antibodies directed against various epitopes of each molecule. The assay for MMP-2 detected pro-MMP-2 (free pro-MMP-2 and pro-MMP-2 complexed with TIMP-2), but not the active form of MMP-2. The assay for MMP-9 recognized pro-MMP-9 (free pro-MMP-9 and pro-MMP-9 complexed with TIMP-1). The assay for TIMP-1 detected free TIMP-1 and TIMP-1 complexed with MMP-9. The assay for TIMP-2 detected free TIMP-2 and TIMP-2 complexed with MMP-2. NGAL and MMP-9/NGAL complex levels were determined with a solid-phase immunoassay, using a commercial kit (Quantikine) from R\&D Systems (Minneapolis, MN, USA). The NGAL assay used two monoclonal antibodies specific for two epitopes of lipocalin 2. The MMP-9/NGAL complex assay used monoclonal antibodies against recombinant human NGAL, and was therefore unable to detect recombinant human MMP-9 or NGAL in their free forms. All assays were conducted according to the manufacturer's instructions. 
Table I. Clinical characteristic of 41 patients.

Characteristic

Patients, n (\%)

Histological grade

Low grade (G1)

High grade (G3)

Tumor stage

Ta

$\mathrm{T} 1$

T2 or higher

Gender

Female

Male

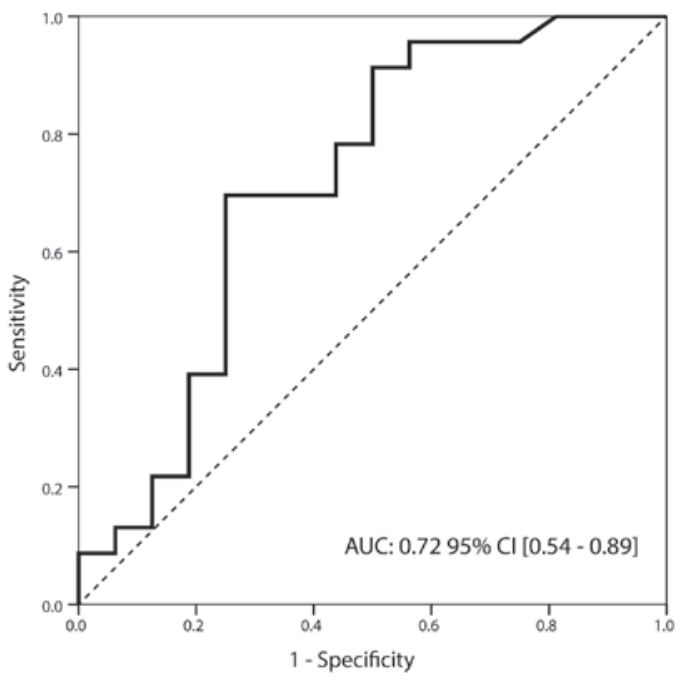

Figure 1. ROC analysis of urinary tissue inhibitor of metalloproteinase-1 expression in discriminating between low- and high-grade bladder cancer. AUC, area under ROC curve; CI, confidence interval; ROC, receiver operating curve.

Statistical analysis. Descriptive statistics are provided as the mean \pm standard deviation, or as the median [range] in the case of quantitative variables; and as frequencies (percentage) in the case of qualitative variables. Student's t-test and the Mann-Whitney $\mathrm{U}$ test were used for comparisons of numeric variables between groups. Prognostic validity of the various biomarkers was evaluated by receiver operating curve (ROC) curve analysis. The diagnostic accuracy was measured using the area under the ROC curve (AUC) and the corresponding 95\% confidence interval (CI).

For all analyses, two-sided tests were used, and $\mathrm{P}<0.05$ was considered to indicate a statistically significant difference.

All statistical analyses were performed using statistical computing environment R software (version 3.01; R Foundation for Statistical Computing, Vienna, Austria).

\section{Results}

During a 1-year period, urine and serum samples from a total of 41 patients with bladder cancer were evaluated, and the histopathological characteristics are listed in Table I.

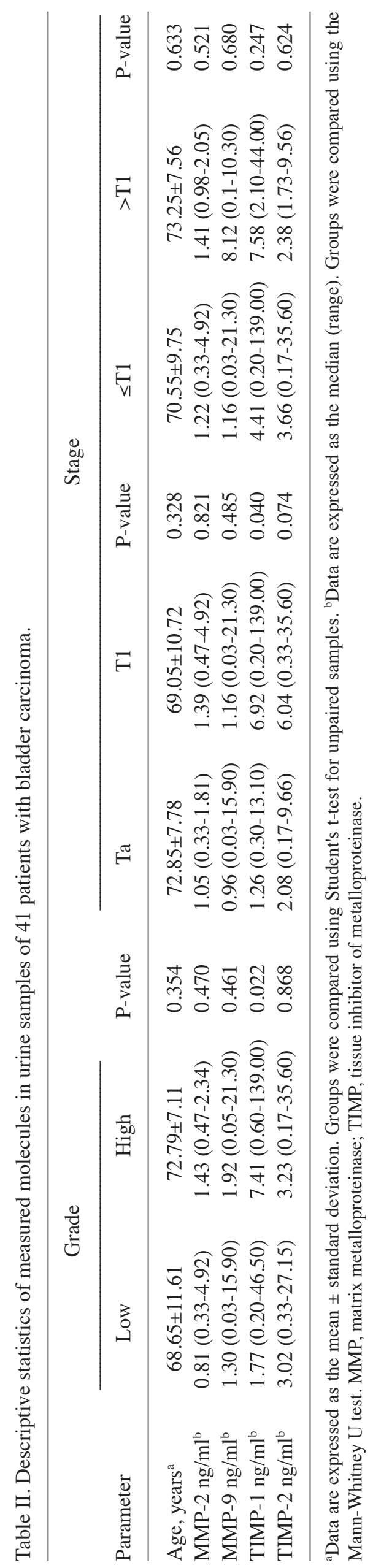




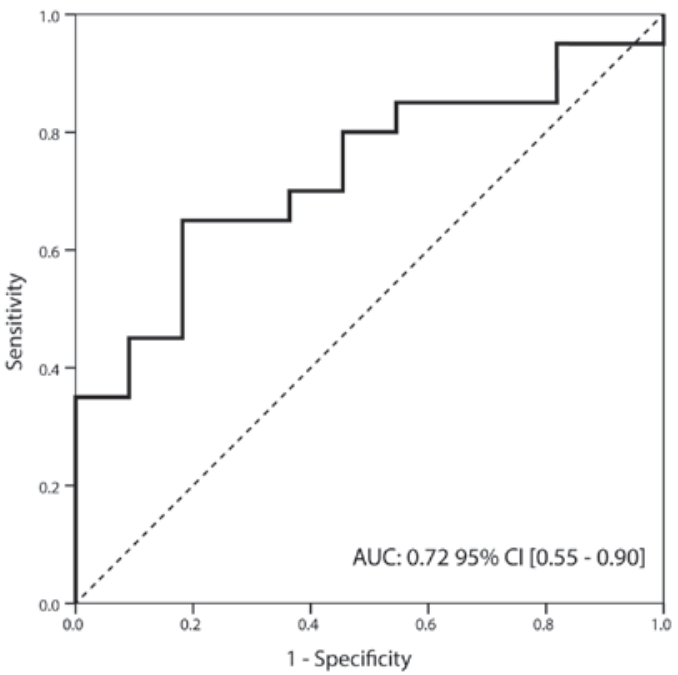

Figure 2. ROC analysis for urinary tissue inhibitor of metalloproteinase-1 in discriminating between Ta and T1 stage bladder cancer. AUC, area under ROC curve; CI, confidence interval; ROC, receiver operating curve.

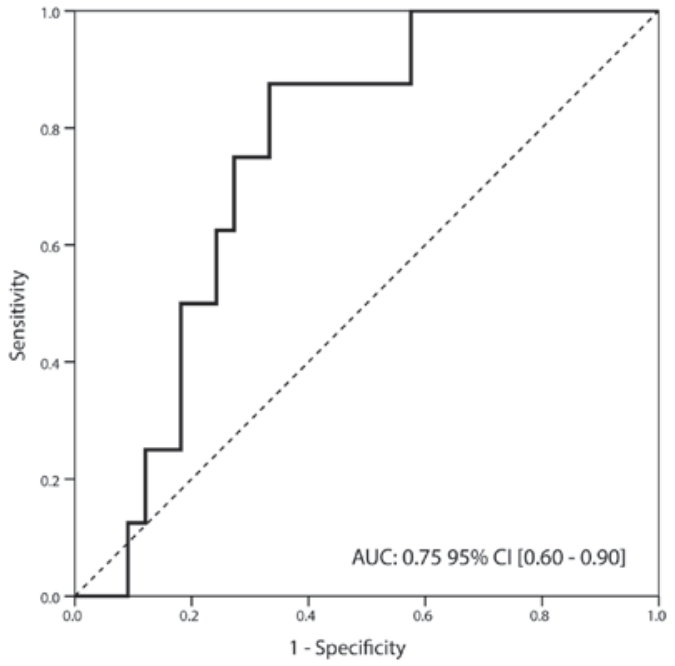

Figure 3. ROC receiver operating curve analysis for serum neutrophil gelatinase-associated lipocalin in discriminating between non-muscle invasive (stage Ta or T1) and muscle invasive (stage $\geq \mathrm{T} 2$ ) bladder cancer. AUC, area under ROC curve; $\mathrm{CI}$, confidence interval; receiver operating curve.

Levels of MMP-2 and -9, as well as their inhibitors TIMP-1 and -2 were determined in urine samples from patients and normal controls. The four molecules were undetectable in the urine samples from all normal controls. By contrast, MMP-2 and -9 were detected in urine samples from 26 of $41(63 \%)$ and 25 of $41(61 \%)$ patients with $\mathrm{BCa}$, respectively. In patients with detectable MMP- 2 and -9 , the median urinary MMP-2 concentration was $1.27 \mathrm{ng} / \mathrm{ml}$, and the median urinary MMP-9 concentration was $1.30 \mathrm{ng} / \mathrm{ml}$. By contrast, TIMP-1 and TIMP-2 were detectable in the majority of urine samples from patients with $\mathrm{BCa}$ analyzed (95 and 83\%, respectively), and the median values were 5.48 and $3.13 \mathrm{ng} / \mathrm{ml}$, respectively. The specimens were divided into histological groups according to grade, low grade (G1) and high grade (G3); and according to $\mathrm{T}$ category, Ta, T1 and $\mathrm{T} 2$ or higher (Tables II and III). 
As shown in Table II, urinary TIMP-1 values were significantly higher in the G3 group than those of the low-grade specimens (7.41 [0.6-139] ng/ml vs. 1.77 [0.2-46.5] ng/ml; $\mathrm{P}=0.022$ ). The corresponding AUC was 0.72 (95\% CI,0.54-0.89), with a sensitivity of 0.70 and a specificity of 0.75 at a cut-off value of $4.95 \mathrm{ng} / \mathrm{ml}$ (Fig. 1). Furthermore, TIMP-1 also demonstrated significantly different expression between $\mathrm{Ta}$ and $\mathrm{T} 1$ stage specimens $(1.26$ [0.3-13.1] $\mathrm{ng} / \mathrm{ml} \mathrm{vs}$. 6.92 [0.2-139] ng/ml; P=0.040). The corresponding AUC was 0.72 (95\% CI, 0.55-0.90) with a sensitivity and specificity of 0.70 and 0.75 at a cut-off value of $4.95 \mathrm{ng} / \mathrm{ml}$, respectively (Fig. 2). Conversely, no significant difference was identified in urinary TIMP-2 concentration between low- and high-grade specimens, or among various tumor stages.

The expression of MMP-2 and -9, their inhibitors TIMP-1 and -2, NGAL and the MMP-9/NGAL complex were determined in sera samples. These six molecules were detected in the sera of all patients studied. In particular, tumors staged as non-muscle invasive (Ta and T1) exhibited significantly higher NGAL levels compared with those of muscle invasive $\mathrm{BCa}$ (32.8 [7.2-126] ng/ml vs. 16.2 [11.2-39] ng/ml; $\mathrm{P}=0.029)$. The discriminatory ability of NGAL expression was confirmed by ROC curve analysis that revealed an AUC of $0.75,(95 \% \mathrm{CI}$, $0.60-0.90)$ and a sensitivity and specificity of 0.88 and 0.67 at a cut-off value of $\leq 26.1 \mathrm{ng} / \mathrm{ml}$, respectively (Fig. 3).

\section{Discussion}

In $\mathrm{BCa}$, the recurrence of treated tumors and the progression of tumors to a higher stage and grade represents a significant risk. Tumor stage and histological grade are strong prognostic factors and provide information useful for the selection of appropriate therapeutic modalities. However, clinical staging is currently imperfect, resulting in significant discrepancies between clinical and pathological stage (20). A potential strategy for the improvement of this limitation is the identification of biomarkers in urine or serum samples, whose levels are indicative of tumor forms and are able to be used to monitor disease progression. The current study aimed to detect biomarkers in the urine and serum of patients with bladder cancer, to differentiate between low- and high-grade tumors and/or among T stages. MMPs and their inhibitors have frequently been investigated in human bladder tissues, and their elevated expression in $\mathrm{BCa}$ tissue at the messenger RNA (mRNA) and protein levels have previously been associated with advanced tumor stage, grade and decreased survival rate (21). Studies have reported that TIMP-1 is a multifunctional protein with a variety of functions independent of MMP inhibition (22). In particular, it has been demonstrated that TIMP-1 is able to stimulate growth in a wide range of cell lines, including fibroblasts and epithelial cells, and is correlated with tumor cell proliferation and angiogenesis (23). By immunohistochemical analysis, Miyata et al (24) revealed that TIMP-1 expression was associated with high T stage and poor prognosis in transitional cell carcinoma of the upper urinary tract. The results of the present study demonstrated that urinary TIMP-1 values were able to differentiate between low- and high-grade $\mathrm{BCa}$, and between Ta and T1 stage. To the best of our knowledge, there are currently few publications available regarding the detection of TIMP-1 in urine for $\mathrm{BCa}$ diagnosis, and these have reported variable results. In particular, Durkan et al (25) found that urinary TIMP-1 values were significantly higher in patients with cancer than those of normal volunteers, with higher values in samples from patients with T2-T4 tumors than those of patients with cis Ta/T1 tumors; but identified no significant variation in expression with tumor grade. By contrast, Monier et al (26) reported that the median value of urinary TIMP-1 was significantly lower in T1-T4 stage patients than that observed in Ta stage specimens.

Furthermore, the present study identified an association between NGAL serum levels and BCa stage. Muscle-invasive $\mathrm{BCa}(>\mathrm{T} 1)$ was able to be differentiated from non-muscle invasive $\mathrm{BCa}(\leq \mathrm{T} 1)$. Until now, NGAL has been investigated primarily as an inflammatory factor and marker of kidney damage (15). However, recent studies have indicated that NGAL has potential role in cancer development and that it may have pro-oncogenic or anti-oncogenic functions. In particular, it has been reported that the NGAL mRNA transcript and protein levels were higher in bladder cancer tissues than those in their normal counterparts (27). Accordingly, $50.5 \%$ of cases displayed NGAL transcript levels higher than the $75^{\text {th }}$ percentile of the 'normal' values, suggesting its role as a diagnostic marker (27). These are in agreement with the results of previous investigations in which NGAL and MMP-9 were overexpressed in urothelial bladder carcinomas, suggesting their role as early diagnostic markers for this tumor type (14). Monier et al (26) found reduced protein levels of NGAL in urine samples from patients with $\mathrm{BCa}$, suggesting that reduced levels of this protein may be used as an indicator of tumor progression. To the best of our knowledge there have been no previous studies regarding the detection of NGAL in the serum of patients with bladder carcinomas. Therefore the present association between sera NGAL and bladder tumor stage has not previously been reported.

In conclusion, taking these observations together, it was suggested that the determination of urinary TIMP-1 and serum NGAL may provide clinicians with additional quantitative and objective information regarding the differentiation of bladder tumors. However, due to the small sample size of the present study, these conclusions may not be transferable to the general population and therefore require further evaluation to validate their diagnostic potential.

\section{References}

1. Ferlay J, Shin HR, Bray F, Forman D, Mathers C and Parkin DM: GLOBOCAN 2008, Cancer incidence and mortality worldwide: IARC CancerBase No. 10. Lyon, France: International Agency for Research on Cancer; 2010.

2. Freedman ND, Silverman DT, Hollenbeck AR, Schatzkin A and Abnet CC: Association between smoking and risk of bladder cancer among men and women. JAMA 306: 737-745, 2011.

3. Burger M, Catto JW, Dalbagni G, Grossman HB, Herr H, Karakiewicz P, Kassouf W, Kiemeney LA, La Vecchia C, Shariat S and Lotan Y: Epidemiology and risk factors of urothelial bladder cancer. Eur Urol 63: 234-241, 2013.

4. Wu XR: Urothelial tumorigenesis: A tale of divergent pathways. Nat Rev Cancer 5: 713-725, 2005.

5. Montie JE, Bahnson RR, Cohen SM, Drucker B, Eisenberger MA, El-Galley R, Herr HW, Hudes GR, Kuzel TM, Lange PH, et al; National Comprehensive Cancer Network: Bladder cancer. Clinical practice guidelines in oncology. J Natl Compr Canc Netw 1: 19-34, 2005. 
6. Carlo A, Terracciano D, Mariano A, Oliva A, D'Armiento M and Macchia V: Role of cytokeratins, nuclear matrix proteins, Lewis antigen and epidermal growth factor receptor in human bladder tumors. Int J Oncol 23: 757-762, 2003.

7. Vrooman OP and Witjes JA: Urinary marker in bladder cancer. Eur Urol 53: 909-916, 2008.

8. Van Tilborg AA, Bangma $\mathrm{CH}$ and Zwarthoff EC: Bladder cancer biomarkers and their role in surveillance and screening. Int $\mathbf{J}$ Urol 16: 23-30, 2009

9. Shariat SF, Passoni N, Bagrodia A, Rachakonda V, Xylinas E, Robinson B, Kapur P, Sagalowsky AI and Lotan Y: Prospective evaluation of a preoperative biomarker panel for prediction of upstaging at radical cystectomy. BJU Int 113: 70-76, 2014.

10. Brinckerhoff CE and Matrisian LM: Matrix metalloproteinases: A tail of a frog that became a prince. Nat Rev Mol Cell Biol 3: 207-214, 2002

11. Di Carlo A, Mariano A, Terracciano D, Mazzarella C, Galzerano S, Cicalese M, Cecere C and Macchia V: Gelatinolytic activities (matrix metalloproteinase-2 and -9) and soluble extracellular domain of Her-2/neu in pleural effusions. Oncol Rep 18: 425-431, 2007.

12. Nagase H, Visse R and Murphy G: Structure and function of matrix metalloproteinases and TIMPs. Cardiovasc Res 69: 562-573, 2006.

13. Di Carlo A, Terracciano D, Mariano A and Macchia V: Urinary gelatinase activities (matrix metalloproteinases 2 and 9) in human bladder tumors. Oncol Rep 15: 1321-1326, 2006.

14. Roy R, Louis G, Loughlin KR, Wiederschain D, Kilroy SM, Lamb CC, Zurakowski D and Moses MA: Tumor-specific urinary matrix metalloproteinase fingerprinting: Identification of high molecular weight urinary matrix metalloproteinase species. Clin Cancer Res 14: 6610-6617, 2008.

15. Chakrabotory S, Kaur S, Guha S and Batra SK. The multifaceted roles of neutrophil gelatinase associated lipocalin (NGAL) in inflammation and cancer. Biochem Biophys Acta 1826: 129-169, 2012.

16. Di Carlo A: Evaluation of neutrophil gelatinase-associated lipocalin (NGAL), matrix metalloproteinase-9 (MMP-9) and their complex MMP-9/NGAL in sera and urine of patients with kidney tumors. Oncol Lett 5: 1677-1681, 2013.

17. Volpe V, Raia Z, Sanguigno L, Somma D, Mastrovito P, Moscato F, Mellone S, Leonardi A and Pacifico F: NGAL controls the metastatic potential of anaplastic thyroid carcinoma cells. J Endocrinol Metab 98: 228-235, 2013.
18. Bouchet $\mathrm{S}$ and Bauvois B: Neutrophil gelatinase-associated lipocalin (NGAL), pro-matrix metalloproteinase-9 (pro-MMP-9) and their complex Pro-MMP-9/NGAL in leukemias. Cancers (Basel) 6: 796-812, 2014

19. Hoboken NJ: Urological tumours. In: TNM Classification of Malignant Tumors. Sobin LH, Gospodariwicz M and Wittekind C (eds). 7th edition. Wiley-Blackwell, London, pp262-265, 2009.

20. Shariat SF, Palapattu GS, Karakiewicz PI, Roger CG, Vazina A, Bastian PJ, Schoenberg MP, Lerner SP, Sagalowsky AI and Lotan Y: Discrepancy between clinical and pathological stage: Impact on prognosis after radical cystectomy. Eur Urol 51: $137-149,2007$

21. Szarvas T, vom Dorp F, Ergün S and Rübben H: Matrix metalloproteinases and their clinical relevance in urinary bladder cancer. Nat Rev Urol 8: 241-254, 2011.

22. Gomez DE, Alonso DF, Yoshiji H and Thorgeirsson UP: Tissue inhibitors of metalloproteinases: Structure, regulation and biological functions. Eur J Cell Biol 74: 111-122, 1997.

23. Hayakawa T: Tissue inhibitors of metalloproteinases and their cell growth-promoting activity. Cell Struct Funct 19: 109-114, 1994.

24. Miyata Y, Kanda S, Nomata K, Hayashida Y and Kanetake H: Expression of metalloproteinase-2, metalloproteinase-9 and tissue inhibitor of metalloproteinase-1 in transitional cell carcinoma of upper urinary tract: Correlation with tumor stage and survival. Urology 63: 602-608, 2004.

25. Durkan GC, Nutt JE, Rajjayabun PH, Neal DE, Lunec J and Mellon JK: Prognostic significance of matrix metalloproteinase- 1 and tissue inhibitor of metalloproteinase- 1 in voided urine samples from patients with transitional cell carcinoma of the bladder. Clinical Cancer Res 7: 3450-3456, 2001.

26. Monier F, Mollier S, Guillot M, Rambeaud JJ, Morel F and Zaoui P: Urinary release of 72 and $92 \mathrm{kDa}$ gelatinases, TIMPs, N-GAL and conventional prognostic factors in urothelial carcinomas. Eur Urol 42: 356-363, 2002.

27. Candido S, Maestro R, Polesel J, Catania A, Maira F, Signorelli SS, McCubrey JA and Libra M: Role of neutrophil gelatinase-associated lipocalin (NGAL) in human cancer. Oncotarget 5: 1576-1594, 2014. 